\title{
The effect of substituting oxygen for terminal nitrogen in aniline oligomers: a DFT comparison of hydroxyl and amino terminated aniline trimers
}

\author{
by \\ Lawrence T. Sein, Jr. \\ Department of Chemical and Physical Sciences, Cedar Crest College, Allentown, PA 18104
}

Email: ltsein@cedarcrest.edu

Phone: 610-606-4666 x3615 


\section{Supplementary Information}

B3LYP/6-31G* optimized structure of syn emeraldine hydroxyl terminated trimer. Cartesian coordinates in Ångstroms.

$\begin{array}{llll}\mathrm{C} & 4.7598614486 & -2.7892617691 & -1.618364884 \\ \mathrm{C} & 4.2989589254 & -1.8394477645 & -0.6997963846 \\ \mathrm{C} & 2.9895809343 & -1.8956478277 & -0.2381685962 \\ \mathrm{C} & 2.1053909051 & -2.9106293008 & -0.6689195529 \\ \mathrm{C} & 2.6081713216 & -3.8877316794 & -1.5512870957 \\ \mathrm{C} & 3.9054375581 & -3.8156726457 & -2.0423603574 \\ \mathrm{~N} & 0.807433051 & -3.0848591229 & -0.1955370629 \\ \mathrm{C} & -0.0257110486 & -2.1039942705 & 0.0195796623 \\ \mathrm{C} & 0.1312318135 & -0.7098700079 & -0.4027683261 \\ \mathrm{C} & -0.7866239431 & 0.2349591872 & -0.0940288486 \\ \mathrm{C} & -1.9893101505 & -0.0827183678 & 0.6800266667 \\ \mathrm{C} & -2.2020139303 & -1.4947955084 & 0.9860231217 \\ \mathrm{C} & -1.2858964888 & -2.4378427058 & 0.6778705005 \\ \mathrm{O} & 6.0521210722 & -2.6766992973 & -2.0484132611 \\ \mathrm{~N} & -2.9192752852 & 0.7510296911 & 1.057639094 \\ \mathrm{H} & 4.9828962961 & -1.0716208193 & -0.3517795294 \\ \mathrm{H} & 2.6556169613 & -1.176740397 & 0.5034657834 \\ \mathrm{H} & 1.9473959423 & -4.6932265625 & -1.855998114 \\ \mathrm{H} & 4.2647766838 & -4.5681347159 & -2.7424095542 \\ \mathrm{H} & 0.9768671732 & -0.4593819156 & -1.0351124473 \\ \mathrm{H} & -0.6777514818 & 1.2439390046 & -0.4784768579 \\ \mathrm{H} & -3.125328846 & -1.7393998383 & 1.5021481845 \\ \mathrm{H} & -1.4312998112 & -3.4832095765 & 0.932415654 \\ \mathrm{H} & -0.7479341288 & 2.3271005077 & 1.6474347238 \\ \mathrm{H} & 6.2461014767 & -3.4041609996 & -2.6598682416 \\ \mathrm{C} & -2.7977639687 & 2.1360187126 & 0.9799929252 \\ \mathrm{C} & -1.6324706892 & 2.8618872008 & 1.3157986238 \\ \mathrm{C} & -1.6184937376 & 4.2510680057 & 1.2897632377 \\ \mathrm{C} & -2.7665622351 & 4.9568409598 & 0.9126744067 \\ \mathrm{C} & -3.9382847998 & 4.2567719611 & 0.5959524016 \\ \mathrm{C} & -3.9571813651 & 2.8692931712 & 0.6571521848 \\ \mathrm{O} & -2.6906879603 & 6.3211351171 & 0.8918170875 \\ \mathrm{H} & -4.8397834194 & 4.8015496454 & 0.3204493981 \\ \mathrm{H} & -4.8672054232 & 2.3201114544 & 0.4368009896 \\ \mathrm{H} & -0.728941851 & 4.8074180667 & 1.5683728669 \\ \mathrm{H} & -3.5539021266 & 6.6817115049 & 0.6357742031\end{array}$


B3LYP/6-31G* optimized structure of syn emeraldine hydroxyl terminated trimer, monohydrochloride salt.

Cartesian coordinates in Ångstroms.

$\begin{array}{llll}\mathrm{C} & 5.3024034999 & -2.835937302 & -1.980498695 \\ \mathrm{C} & 4.8508768525 & -1.8226831018 & -1.1223921846 \\ \mathrm{C} & 3.5527215221 & -1.8518671191 & -0.6373906313 \\ \mathrm{C} & 2.668058977 & -2.9048071901 & -0.9814452972 \\ \mathrm{C} & 3.1683473873 & -3.9491916738 & -1.7917423133 \\ \mathrm{C} & 4.4519924423 & -3.902392572 & -2.3117546951 \\ \mathrm{~N} & 1.3799637963 & -3.0547418307 & -0.5037556707 \\ \mathrm{C} & 0.5599897383 & -2.0646498666 & -0.2516872961 \\ \mathrm{C} & 0.7121355914 & -0.6776684609 & -0.680169773 \\ \mathrm{C} & -0.1891532845 & 0.2743858134 & -0.3322903958 \\ \mathrm{C} & -1.331648985 & -0.0548880419 & 0.4951445272 \\ \mathrm{C} & -1.567595008 & -1.4477061296 & 0.8184600573 \\ \mathrm{C} & -0.6717139802 & -2.3941902801 & 0.4499616441 \\ \mathrm{O} & \mathbf{6 . 5 8 0 0 6 4 6 6 0 5} & -2.7436156148 & -2.4358358667 \\ \mathrm{~N} & -2.2220296859 & 0.8274262825 & 0.9213537344 \\ \mathrm{H} & 5.5395675499 & -1.0337098735 & -0.8377534274 \\ \mathrm{H} & 3.2316077962 & -1.0917025211 & 0.0671668407 \\ \mathrm{H} & 2.5075384912 & -4.7790507368 & -2.020258783 \\ \mathrm{H} & 4.8088809804 & -4.7017323244 & -2.9582487961 \\ \mathrm{H} & 1.5199058755 & -0.4308468731 & -1.3606950617 \\ \mathrm{H} & -0.1049224448 & 1.2788772061 & -0.7310484938 \\ \mathrm{H} & -2.4770570049 & -1.6859016668 & 1.3645105009 \\ \mathrm{H} & -0.8196121715 & -3.4390057716 & 0.7030438884 \\ \mathrm{H} & -0.0633974427 & 2.5166983788 & 1.1095460709 \\ \mathrm{H} & 6.781552881 & -3.5116548415 & -2.9937807526 \\ \mathrm{C} & -2.1990092404 & 2.2278017809 & 0.8704222002 \\ \mathrm{C} & -1.0241323529 & 2.9987790188 & 0.966693198 \\ \mathrm{C} & -1.0929210642 & 4.3840547915 & 0.948971146 \\ \mathrm{C} & -2.336626873 & 5.0235921921 & 0.8465448838 \\ \mathrm{C} & -3.512007402 & 4.2617117766 & 0.7891947799 \\ \mathrm{C} & -3.4478482967 & 2.8763311722 & 0.8124174775 \\ \mathrm{O} & -2.3369746314 & 6.3833852809 & 0.8271531772 \\ \mathrm{H} & -4.4799114839 & 4.7555294994 & 0.7342910035 \\ \mathrm{H} & -4.3498692443 & 2.2710759125 & 0.7947840817 \\ \mathrm{H} & -0.1972988809 & 4.9899567903 & 1.0378904346 \\ \mathrm{H} & -3.2520020574 & 6.7045981174 & 0.7889438291 \\ \mathrm{Cl} & -4.7581940449 & -0.2716582651 & 1.8512773299 \\ \mathrm{H} & -3.1691602281 & 0.4040618728 & 1.3255373299\end{array}$


B3LYP/6-31G* optimized structure of syn emeraldine hydroxyl terminated trimer, dihydrochloride salt.

Cartesian coordinates in Ångstroms.

$\begin{array}{llll}\mathrm{C} & 5.290545856 & -2.3078675097 & -2.0353182575 \\ \mathrm{C} & 4.825956654 & -1.2002714057 & -1.3093750651 \\ \mathrm{C} & 3.5415890939 & -1.2020003877 & -0.7888328968 \\ \mathrm{C} & 2.6928854123 & -2.3092450995 & -0.9933364643 \\ \mathrm{C} & 3.1818562591 & -3.4368251334 & -1.6841025815 \\ \mathrm{C} & 4.4643223743 & -3.4277449113 & -2.2099882572 \\ \mathrm{~N} & 1.4003795821 & -2.4099488516 & -0.4693076718 \\ \mathrm{C} & 0.505893273 & -1.4660202976 & -0.2255356387 \\ \mathrm{C} & 0.6201113591 & -0.0957670346 & -0.6816186251 \\ \mathrm{C} & -0.3106503699 & 0.8279511407 & -0.33759362 \\ \mathrm{C} & -1.4598139663 & 0.4772158953 & 0.4885000256 \\ \mathrm{C} & -1.6344619052 & -0.9317431421 & 0.8052242899 \\ \mathrm{C} & -0.7007088918 & -1.8564651369 & 0.4769452024 \\ \mathrm{O} & 6.5550492106 & -2.2412774206 & -2.5247741986 \\ \mathrm{~N} & -2.38375258 & 1.3155113195 & 0.9002447212 \\ \mathrm{H} & 5.4962104354 & -0.3633421853 & -1.1442624131 \\ \mathrm{H} & 3.216470533 & -0.3697034432 & -0.174568224 \\ \mathrm{H} & 2.5371334565 & -4.3047782673 & -1.7891985332 \\ \mathrm{H} & 4.8309320063 & -4.296610052 & -2.7522803589 \\ \mathrm{H} & 1.41875051 & 0.1635030829 & -1.3672035731 \\ \mathrm{H} & -0.2554925565 & 1.8298133328 & -0.7487738444 \\ \mathrm{H} & -2.5266798702 & -1.2149473428 & 1.3542555068 \\ \mathrm{H} & -0.8114915573 & -2.9071116351 & 0.7333959028 \\ \mathrm{H} & -0.1738562451 & 2.8847675335 & 1.3319881494 \\ \mathrm{H} & 6.7735327684 & -3.0734480015 & -2.9743236406 \\ \mathrm{C} & -2.2835932591 & 2.6992552011 & 0.8652323732 \\ \mathrm{C} & -1.0867851316 & 3.4208044206 & 1.0944358518 \\ \mathrm{C} & -1.0815906922 & 4.8060698567 & 1.0996943457 \\ \mathrm{C} & -2.2713324625 & 5.5127799768 & 0.8676168302 \\ \mathrm{C} & -3.4721938107 & 4.8141731471 & 0.6680375245 \\ \mathrm{C} & -3.481163687 & 3.4287925851 & 0.6942482202 \\ \mathrm{O} & -2.1996563125 & 6.8681571159 & 0.8734999197 \\ \mathrm{H} & -4.4000814102 & 5.3598888534 & 0.5105802027 \\ \mathrm{H} & -4.4106151479 & 2.8853222997 & 0.5606401563 \\ \mathrm{H} & -0.1730492523 & 5.3635520934 & 1.3026114614 \\ \mathrm{H} & -3.084829775 & 7.2425543379 & 0.7374410954 \\ \mathrm{Cl} & -5.0609911419 & 0.2993956743 & 2.0427438991 \\ \mathrm{H} & -3.8727702085 & 0.7211065628 & 1.5581446474 \\ \mathrm{H} & 1.0607445311 & -3.4684246509 & -0.2878712064 \\ \mathrm{Cl} & 0.4945419037 & -5.1420233416 & -0.1264109572 \\ & & & \\ & & \end{array}$


B3L YP/6-31G* optimized structure of syn leucoemeraldine hydroxyl terminated trimer. Cartesian coordinates in Ångstroms.

$\begin{array}{llll}\mathrm{C} & 4.903130072 & -2.8892656432 & -1.5693541323 \\ \mathrm{C} & 4.3705054842 & -1.8914649386 & -0.7473552324 \\ \mathrm{C} & 3.0387859876 & -1.9406455843 & -0.3492648131 \\ \mathrm{C} & 2.1929944932 & -2.978116975 & -0.7835411525 \\ \mathrm{C} & 2.7390180495 & -3.9709052413 & -1.6116493108 \\ \mathrm{C} & 4.0801874842 & -3.9361720718 & -1.9903578265 \\ \mathrm{~N} & 0.8570172399 & -3.0866068287 & -0.3731906765 \\ \mathrm{C} & -0.0484554856 & -2.0645640167 & -0.0530533321 \\ \mathrm{C} & 0.0843934966 & -0.7448721695 & -0.5133167281 \\ \mathrm{C} & -0.8589521463 & 0.2281811417 & -0.1912239201 \\ \mathrm{C} & -1.9744295884 & -0.0781262049 & 0.6043651046 \\ \mathrm{C} & -2.1147779855 & -1.4033250923 & 1.0509070131 \\ \mathrm{C} & -1.1739849857 & -2.3736095259 & 0.7298296015 \\ \mathrm{O} & 6.2261529848 & -2.7870520399 & -1.9214282018 \\ \mathrm{~N} & -2.9664943673 & 0.8572033335 & 0.9332682443 \\ \mathrm{H} & 5.0184469323 & -1.0879624739 & -0.4108138613 \\ \mathrm{H} & 2.6537211065 & -1.1772176176 & 0.318056954 \\ \mathrm{H} & 2.1050661655 & -4.7810104825 & -1.9654154242 \\ \mathrm{H} & 4.4791551734 & -4.7227520689 & -2.6288991525 \\ \mathrm{H} & 0.9155921619 & -0.4798350013 & -1.1577694454 \\ \mathrm{H} & -0.7411290294 & 1.2291325729 & -0.5920214726 \\ \mathrm{H} & -2.9666493208 & -1.6697183599 & 1.6730580352 \\ \mathrm{H} & -1.3017025583 & -3.3869332591 & 1.1047843076 \\ \mathrm{H} & -0.7994291567 & 2.3864378857 & 1.4937241892 \\ \mathrm{H} & 6.4573429914 & -3.5405090689 & -2.4858586061 \\ \mathrm{C} & -2.883997212 & 2.2566366299 & 0.9401070256 \\ \mathrm{C} & -1.6986285889 & 2.9452129378 & 1.2577972985 \\ \mathrm{C} & -1.668440499 & 4.3352453353 & 1.294388205 \\ \mathrm{C} & -2.8258375279 & 5.0773658045 & 1.0401956294 \\ \mathrm{C} & -4.0143077453 & 4.4060002798 & 0.7445163685 \\ \mathrm{C} & -4.0374498952 & 3.0135425543 & 0.6827990969 \\ \mathrm{O} & -2.7320938135 & 6.4457417534 & 1.0987715657 \\ \mathrm{H} & -4.9253674382 & 4.9683359456 & 0.5461064849 \\ \mathrm{H} & -4.9654543177 & 2.5055302531 & 0.429723156 \\ \mathrm{H} & -0.7507949589 & 4.8616883316 & 1.5382723796 \\ \mathrm{H} & -3.6018898167 & 6.8262136449 & 0.9027510321 \\ \mathrm{H} & 0.4677108479 & -4.0176042436 & -0.4020381331 \\ \mathrm{H} & -3.8892727059 & 0.4758073817 & 1.0823163006\end{array}$


B3LYP/6-31G* optimized structure of syn pernigraniline hydroxyl terminated trimer. Cartesian coordinates in Ångstroms.

$\begin{array}{llll}\mathrm{C} & 4.9255081231 & -2.8346700205 & -1.336754593 \\ \mathrm{C} & 4.2122709036 & -1.6832053708 & -0.7360446533 \\ \mathrm{C} & 2.8852392105 & -1.707903759 & -0.5006798004 \\ \mathrm{C} & 2.0805354645 & -2.8937748762 & -0.8256685367 \\ \mathrm{C} & 2.7919398114 & -4.0653433663 & -1.3466889757 \\ \mathrm{C} & 4.1132873438 & -4.0385915624 & -1.6088292868 \\ \mathrm{~N} & 0.7972948688 & -3.0251587667 & -0.6599519546 \\ \mathrm{C} & -0.0634990609 & -1.9840236854 & -0.3331695483 \\ \mathrm{C} & -0.0411886659 & -0.7172466503 & -0.9547363011 \\ \mathrm{C} & -0.9838464757 & 0.250661118 & -0.6300361153 \\ \mathrm{C} & -1.9857313877 & -0.0102981116 & 0.3289539122 \\ \mathrm{C} & -2.0487531125 & -1.3032940233 & 0.8956718123 \\ \mathrm{C} & -1.1108158193 & -2.2663638978 & 0.5725897085 \\ \mathrm{O} & 6.1310653637 & -2.7922233674 & -1.5704110857 \\ \mathrm{~N} & -3.0047924007 & 0.8787616416 & 0.6497178166 \\ \mathrm{H} & 4.8270575553 & -0.8258111186 & -0.4769357118 \\ \mathrm{H} & 2.388822102 & -0.8660823572 & -0.0292548566 \\ \mathrm{H} & 2.1800941517 & -4.9415795401 & -1.5402473248 \\ \mathrm{H} & 4.6398984768 & -4.8928022862 & -2.0237451886 \\ \mathrm{H} & 0.6880610647 & -0.5167040583 & -1.7336187171 \\ \mathrm{H} & -0.9831829954 & 1.1993493084 & -1.157927545 \\ \mathrm{H} & -2.8530792643 & -1.5213019083 & 1.5910660864 \\ \mathrm{H} & -1.1604105197 & -3.2593289814 & 1.0080235871 \\ \mathrm{H} & -0.6375625832 & 2.2415342821 & 1.0128674758 \\ \mathrm{H} & -0.547421394 & 4.6927294423 & 1.3740885825 \\ \mathrm{C} & -2.8309475949 & 2.1493430591 & 0.8661299984 \\ \mathrm{C} & -1.5431221766 & 2.8391620407 & 1.0245287592 \\ \mathrm{C} & -1.4887968151 & 4.1706407254 & 1.2275871466 \\ \mathrm{C} & -2.7106815858 & 5.0061556609 & 1.2936656692 \\ \mathrm{C} & -3.9993797958 & 4.2914953741 & 1.1857516218 \\ \mathrm{C} & -4.0467041887 & 2.9566099035 & 1.00903478 \\ \mathrm{O} & -2.6486284411 & 6.2227673278 & 1.4541950934 \\ \mathrm{H} & -4.8963164939 & 4.8989094504 & 1.2612613559 \\ \mathrm{H} & -4.9848578259 & 2.4154006099 & 0.9279555741\end{array}$


B3LYP/6-31G* optimized structure of anti emeraldine hydroxyl terminated trimer. Cartesian coordinates in Ångstroms.

$\begin{array}{llll}\mathrm{H} & -7.8331421173 & -0.0608258529 & -1.0121180508 \\ \mathrm{H} & -5.7739703405 & -0.8748318741 & -1.6395038531 \\ \mathrm{H} & -2.2836124854 & -0.9121329933 & 0.4828131763 \\ \mathrm{H} & 2.2822474661 & 0.9204283845 & -0.4697363875 \\ \mathrm{H} & -6.4379166789 & 2.7994735616 & 0.4853566824 \\ \mathrm{H} & -3.3395116532 & -0.4860835149 & -1.5737601791 \\ \mathrm{H} & -0.295869521 & -2.3356543691 & 0.807392307 \\ \mathrm{~N} & 2.116400854 & -1.6929257827 & 0.4540724893 \\ \mathrm{C} & 1.1579838689 & -0.8375311388 & 0.2255600309 \\ \mathrm{C} & 1.291922245 & 0.5437119623 & -0.2395040813 \\ \mathrm{C} & 0.204987401 & 1.3254774859 & -0.4258734694 \\ \mathrm{C} & -1.1590608201 & 0.8444377998 & -0.2143443663 \\ \mathrm{C} & -1.2930441435 & -0.536440708 & 0.2517315488 \\ \mathrm{C} & -0.206106847 & -1.3181585755 & 0.4381724219 \\ \mathrm{~N} & -2.1179961475 & 1.6983780236 & -0.4452631624 \\ \mathrm{C} & -3.4710415543 & 1.3658628519 & -0.4638078885 \\ \mathrm{C} & -4.3776614605 & 2.3079699611 & 0.0691743164 \\ \mathrm{C} & -5.7454349302 & 2.0839038838 & 0.0533601956 \\ \mathrm{C} & -6.2567550254 & 0.9273688305 & -0.5516544832 \\ \mathrm{C} & -5.3807330868 & 0.006450194 & -1.1352450738 \\ \mathrm{C} & -4.004983373 & 0.2179780241 & -1.0842809657 \\ \mathrm{O} & -7.6140511752 & 0.7678632312 & -0.558250418 \\ \mathrm{H} & 3.9687395509 & -3.2135353051 & -0.4960661965 \\ \mathrm{H} & -3.973211876 & 3.2122522924 & 0.5132081784 \\ \mathrm{H} & 0.2949974601 & 2.343357527 & -0.7938815154 \\ \mathrm{C} & 3.4698677517 & -1.3618458959 & 0.4705931049 \\ \mathrm{C} & 4.0084087749 & -0.208902398 & 1.0849476454 \\ \mathrm{C} & 5.3803222812 & 0.0065539551 & 1.130775317 \\ \mathrm{C} & 6.2552320126 & -0.9176473773 & 0.5487250029 \\ \mathrm{C} & 5.742481763 & -2.0775133195 & -0.0466625954 \\ \mathrm{C} & 4.3721887461 & -2.3059459082 & -0.0580566639 \\ \mathrm{O} & 7.5932227292 & -0.6459383196 & 0.6100251948 \\ \mathrm{H} & 6.4197124309 & -2.8096166785 & -0.483520339 \\ \mathrm{H} & 8.0821324676 & -1.3696934793 & 0.1883498193 \\ \mathrm{H} & 5.7930127905 & 0.8824885177 & 1.6215236152 \\ \mathrm{H} & 3.3427455074 & 0.4964310404 & 1.5724192607\end{array}$


B3LYP/6-31G* optimized structure of anti emeraldine hydroxyl terminated trimer, monohydrochloride salt.

Cartesian coordinates in Ångstroms.

$\begin{array}{llll}\mathrm{H} & -7.7747033701 & -0.3874121022 & -0.9028192908 \\ \mathrm{H} & -5.7445295925 & -1.3375428494 & -1.4339396051 \\ \mathrm{H} & -2.0763602765 & -1.5186899048 & 0.3589043181 \\ \mathrm{H} & 2.4265841474 & 0.5279230751 & -0.4027354604 \\ \mathrm{H} & -6.276949321 & 2.5151282972 & 0.3999192884 \\ \mathrm{H} & -3.3024723422 & -1.0420626852 & -1.378099412 \\ \mathrm{H} & -0.0476059738 & -2.9108421272 & 0.5472437452 \\ \mathrm{~N} & 2.3403785273 & -2.1658662148 & 0.306777984 \\ \mathrm{C} & 1.3575578914 & -1.3146218495 & 0.1376714248 \\ \mathrm{C} & 1.4518261394 & 0.0995856474 & -0.2004314058 \\ \mathrm{C} & 0.3446058953 & 0.8697294602 & -0.3329723852 \\ \mathrm{C} & -0.9827413284 & 0.3073993632 & -0.1625105823 \\ \mathrm{C} & -1.0984387778 & -1.0958180646 & 0.1654806828 \\ \mathrm{C} & 0.0158838396 & -1.8608384785 & 0.2795939803 \\ \mathrm{~N} & -2.0046908578 & 1.135399661 & -0.3184942796 \\ \mathrm{C} & -3.3817949921 & 0.8754254224 & -0.3709396871 \\ \mathrm{C} & -4.2362330325 & 1.9006083223 & 0.0870072606 \\ \mathrm{C} & -5.6105874444 & 1.7378484871 & 0.0411105353 \\ \mathrm{C} & -6.1616051019 & 0.5605057793 & -0.4853520753 \\ \mathrm{C} & -5.3195359273 & -0.4426535157 & -0.9840351287 \\ \mathrm{C} & -3.9390202551 & -0.2859636021 & -0.9324182957 \\ \mathrm{O} & -7.5178876107 & 0.4583513402 & -0.502627334 \\ \mathrm{H} & 4.2287775597 & -3.6922914196 & -0.479650581 \\ \mathrm{H} & -3.7931944833 & 2.8169548531 & 0.4662557935 \\ \mathrm{H} & 0.4035857458 & 1.9230606956 & -0.59701946 \\ \mathrm{C} & 3.67407464 & -1.8240947492 & 0.4254883343 \\ \mathrm{C} & 4.1745622238 & -0.6587813457 & 1.0602887395 \\ \mathrm{C} & 5.5376768513 & -0.4427668804 & 1.1808942466 \\ \mathrm{C} & 6.4463811625 & -1.3732363606 & 0.6545456923 \\ \mathrm{C} & 5.9731427128 & -2.546339085 & 0.0464962733 \\ \mathrm{C} & 4.6094750771 & -2.7793111118 & -0.0333576345 \\ \mathrm{O} & 7.7697516499 & -1.095579079 & 0.7885209607 \\ \mathrm{H} & \mathbf{6} .6769633796 & -3.2802753357 & -0.3410983784 \\ \mathrm{H} & 8.2931800542 & -1.8164879826 & 0.4036804821 \\ \mathrm{H} & 5.9234489706 & 0.4348297856 & 1.6894151707 \\ \mathrm{H} & 3.4852873415 & 0.0451935145 & 1.5141296509 \\ \mathrm{H} & -1.744592437 & 2.2160450462 & -0.3573104192 \\ \mathrm{Cl} & -1.3043248732 & 3.968087782 & -0.3030218022\end{array}$


B3LYP/6-31G* optimized structure of anti emeraldine hydroxyl terminated trimer, dihydrochloride salt.

Cartesian coordinates in Ångstroms.

$\begin{array}{llll}\mathrm{H} & -7.9261513343 & 0.0164250533 & -1.0474562624 \\ \mathrm{H} & -5.8662010971 & -0.9034291063 & -1.5195188943 \\ \mathrm{H} & -2.2292653074 & -1.0698308485 & 0.3488352886 \\ \mathrm{H} & 2.2276759304 & 1.0976588845 & -0.3317871134 \\ \mathrm{H} & -6.5187604601 & 2.9437336152 & 0.2972076112 \\ \mathrm{H} & -3.4353226941 & -0.581905717 & -1.3691177208 \\ \mathrm{H} & -0.1784553132 & -2.4323378395 & 0.489644436 \\ \mathrm{~N} & 2.2115719418 & -1.5900690251 & 0.2535730511 \\ \mathrm{C} & 1.1759070405 & -0.7696069185 & 0.127734462 \\ \mathrm{C} & 1.2617525111 & 0.6444455007 & -0.1477494551 \\ \mathrm{C} & 0.1359449925 & 1.3958100221 & -0.2508884088 \\ \mathrm{C} & -1.1777218597 & 0.7959346937 & -0.1154794747 \\ \mathrm{C} & -1.2630981029 & -0.6177062513 & 0.1630839664 \\ \mathrm{C} & -0.13752502 & -1.369094121 & 0.2656784265 \\ \mathrm{~N} & -2.2150669838 & 1.6129068379 & -0.2466252896 \\ \mathrm{C} & -3.5744584144 & 1.3375749127 & -0.3615668846 \\ \mathrm{C} & -4.4610994847 & 2.3575765684 & 0.0593704623 \\ \mathrm{C} & -5.8277367414 & 2.1771769072 & -0.0364802161 \\ \mathrm{C} & -6.3419022753 & 0.9891529733 & -0.5820373558 \\ \mathrm{C} & -5.4680048997 & -0.0066620501 & -1.050195865 \\ \mathrm{C} & -4.0965329925 & 0.1640984261 & -0.944054764 \\ \mathrm{O} & -7.6884327423 & 0.8702574354 & -0.6511018347 \\ \mathrm{H} & 4.0151391606 & -3.2817096974 & -0.4315212789 \\ \mathrm{H} & -4.0433754769 & 3.2767240734 & 0.4598964502 \\ \mathrm{H} & 0.1769003944 & 2.4592348664 & -0.474073402 \\ \mathrm{C} & 3.5734950462 & -1.3266122882 & 0.3643921155 \\ \mathrm{C} & 4.1134405508 & -0.1498327169 & 0.9304597896 \\ \mathrm{C} & 5.48389774 & 0.0064797918 & 1.0338467931 \\ \mathrm{C} & 6.344763391 & -1.0084855918 & 0.5804819411 \\ \mathrm{C} & 5.8149600111 & -2.1960040238 & 0.0512418606 \\ \mathrm{C} & 4.4440063873 & -2.3621327088 & -0.0435977954 \\ \mathrm{O} & 7.6742216702 & -0.7867161766 & 0.7022756901 \\ \mathrm{H} & 6.4800766583 & -2.9908343518 & -0.2785601053 \\ \mathrm{H} & 8.1678390116 & -1.5606107354 & 0.3853641138 \\ \mathrm{H} & 5.9137420753 & 0.8946753773 & 1.4843252194 \\ \mathrm{H} & 3.4616178979 & 0.6105064894 & 1.3443298177 \\ \mathrm{H} & -1.9661059158 & 2.7137511709 & -0.2187454914 \\ \mathrm{H} & 1.9577098968 & -2.6844518344 & 0.2233345928 \\ \mathrm{Cl} & -1.551984288 & 4.4267940343 & -0.0826577747 \\ \mathrm{Cl} & 1.5461350624 & -4.4096453821 & 0.0776213029\end{array}$


B3LYP/6-31G* optimized structure of anti leucoemeraldine hydroxyl terminated trimer. Cartesian coordinates in Ångstroms.

$\begin{array}{llll}\mathrm{C} & 1.7413257731 & -6.1102516473 & -1.1401655463 \\ \mathrm{C} & 0.6030349597 & -5.3369836649 & -1.3879965584 \\ \mathrm{C} & 0.6510518841 & -3.9520948554 & -1.2689347323 \\ \mathrm{C} & 1.8341127025 & -3.3013469883 & -0.8724838129 \\ \mathrm{C} & 2.9681821865 & -4.0894699279 & -0.6234475276 \\ \mathrm{C} & 2.9283200443 & -5.4755207092 & -0.7678684441 \\ \mathrm{~N} & 1.9325178025 & -1.9058671695 & -0.7826099679 \\ \mathrm{C} & 0.946289381 & -0.983617602 & -0.3998601763 \\ \mathrm{C} & 1.1106844503 & 0.3680428703 & -0.7442496114 \\ \mathrm{C} & 0.1872816896 & 1.3354838248 & -0.3615301541 \\ \mathrm{C} & -0.9485518248 & 0.9848877488 & 0.3869359966 \\ \mathrm{C} & -1.1129449378 & -0.3667542725 & 0.7312496091 \\ \mathrm{C} & -0.1894982558 & -1.3342315376 & 0.3484853659 \\ \mathrm{O} & 1.6310968741 & -7.4714172298 & -1.2812663051 \\ \mathrm{~N} & -1.9348797066 & 1.9072372145 & 0.7682685452 \\ \mathrm{H} & -0.3127822425 & -5.8342153414 & -1.6925434515 \\ \mathrm{H} & -0.2312675112 & -3.3658888526 & -1.5022798004 \\ \mathrm{H} & 3.8941835087 & -3.6116703024 & -0.3111853574 \\ \mathrm{H} & 3.8247581757 & -6.0623859396 & -0.573928942 \\ \mathrm{H} & 1.9710331355 & 0.6631825674 & -1.3413898212 \\ \mathrm{H} & 0.3354231564 & 2.3639736051 & -0.6721954918 \\ \mathrm{H} & -1.9733595349 & -0.6620249308 & 1.3282452139 \\ \mathrm{H} & -0.3378049974 & -2.3627357379 & 0.6589926698 \\ \mathrm{H} & 0.2329303483 & 3.3611369148 & 1.490822617 \\ \mathrm{H} & 2.4880319244 & -7.8759327429 & -1.0767908875 \\ \mathrm{C} & -1.8347482911 & 3.3018545141 & 0.8679100363 \\ \mathrm{C} & -0.6498685456 & 3.9492655929 & 1.2643275037 \\ \mathrm{C} & -0.6005609576 & 5.3333666314 & 1.3920980906 \\ \mathrm{C} & -1.7391408555 & 6.1089574533 & 1.1532216095 \\ \mathrm{C} & -2.9278696893 & 5.4773779212 & 0.7810160839 \\ \mathrm{C} & -2.9691749319 & 4.0923436632 & 0.627878372 \\ \mathrm{O} & -1.6275375313 & 7.469142743 & 1.3026677558 \\ \mathrm{H} & -3.8245938915 & 6.0660531324 & 0.5940015103 \\ \mathrm{H} & -3.8965191158 & 3.6170936759 & 0.3157330055 \\ \mathrm{H} & 0.3166651856 & 5.82802968 & 1.6965970131 \\ \mathrm{H} & -2.4847795218 & 7.8755252425 & 1.1032490939 \\ \mathrm{H} & -2.8678764417 & 1.5314603732 & 0.8552388041 \\ \mathrm{H} & 2.8664677196 & -1.5308498526 & -0.8629044478\end{array}$


B3LYP/6-31G* optimized structure of anti pernigraniline hydroxyl terminated trimer. Cartesian coordinates in Ångstroms.

$\begin{array}{llcc}\mathrm{H} & 3.1714100523 & 0.5551955271 & 1.1497333436 \\ \mathrm{H} & -5.5845743747 & -0.9377903778 & -1.5659438847 \\ \mathrm{H} & -2.1021177871 & -0.8599666159 & 1.0031444884 \\ \mathrm{H} & 2.102020734 & 0.8598290271 & -1.0029648356 \\ \mathrm{H} & -6.5283162467 & 2.9529809093 & 0.1490873593 \\ \mathrm{H} & -3.1713517191 & -0.5551967677 & -1.1495829626 \\ \mathrm{H} & -0.0983110967 & -2.3157547136 & 0.8836678207 \\ \mathrm{~N} & 2.218474751 & -1.7136801486 & -0.0902689456 \\ \mathrm{C} & 1.1525613703 & -0.8231274365 & -0.0259678735 \\ \mathrm{C} & 1.1906552982 & 0.4927559332 & -0.5413033334 \\ \mathrm{C} & 0.0665043786 & 1.3024612601 & -0.4946245239 \\ \mathrm{C} & -1.1526008455 & 0.8230049033 & 0.0262429555 \\ \mathrm{C} & -1.1907147216 & -0.4929062973 & 0.5415479342 \\ \mathrm{C} & -0.0665804236 & -1.3025935471 & 0.4948718357 \\ \mathrm{~N} & -2.2185344648 & 1.7135590543 & 0.0906817327 \\ \mathrm{C} & -3.4495679801 & 1.4087769465 & -0.1963140768 \\ \mathrm{C} & -4.4594126654 & 2.4279828589 & 0.1073185761 \\ \mathrm{C} & -5.773075063 & 2.2140014586 & -0.1013774887 \\ \mathrm{C} & -6.2603988053 & 0.9511954559 & -0.6942571371 \\ \mathrm{C} & -5.2255994275 & -0.0403916142 & -1.0699589956 \\ \mathrm{C} & -3.9150058948 & 0.1695306801 & -0.834820001 \\ \mathrm{O} & -7.4546732448 & 0.7391025024 & -0.8902232744 \\ \mathrm{H} & 4.083913818 & -3.3500071171 & -0.5414495772 \\ \mathrm{H} & -4.0839709648 & 3.3499994308 & 0.5415665633 \\ \mathrm{H} & 0.0982605195 & 2.3156074291 & -0.883455413 \\ \mathrm{C} & 3.4495454194 & -1.4088463813 & 0.1965927888 \\ \mathrm{C} & 3.9150418276 & -0.1695147833 & 0.8348831263 \\ \mathrm{C} & 5.225672268 & 0.0404873081 & 1.0697425001 \\ \mathrm{C} & 6.2604535001 & -0.9510688767 & 0.6938873996 \\ \mathrm{C} & 5.7730973336 & -2.2138737986 & 0.1010620722 \\ \mathrm{C} & 4.4593940111 & -2.4279586385 & -0.1073023524 \\ \mathrm{O} & 7.4547465804 & -0.7389309014 & 0.8897122895 \\ \mathrm{H} & 6.5283161876 & -2.9528151223 & -0.1495819316 \\ \mathrm{H} & 5.5847347086 & 0.937900655 & 1.5656369627 \\ & & & \end{array}$


B3LYP/6-31G* optimized structure of syn emeraldine hydroxyl terminated trimer in acetonitrile solvent.

Cartesian coordinates in Ångstroms.

$\begin{array}{lrcc}\mathrm{C} & 5.541101 & -1.518447 & 0.102760 \\ \mathrm{C} & 4.361599 & -1.711750 & -0.631179 \\ \mathrm{C} & 3.409576 & -0.701484 & -0.706152 \\ \mathrm{C} & 3.614163 & 0.540814 & -0.063095 \\ \mathrm{C} & 4.831016 & 0.734162 & 0.623787 \\ \mathrm{C} & 5.769119 & -0.284921 & 0.732348 \\ \mathrm{~N} & 2.749171 & 1.632261 & -0.157758 \\ \mathrm{C} & 1.446462 & 1.530964 & -0.101860 \\ \mathrm{C} & 0.676024 & 0.353872 & 0.303576 \\ \mathrm{C} & -0.678004 & 0.354659 & 0.304825 \\ \mathrm{C} & -1.448109 & 1.532478 & -0.099308 \\ \mathrm{C} & -0.676281 & 2.738262 & -0.387753 \\ \mathrm{C} & 0.675225 & 2.737457 & -0.389171 \\ \mathrm{O} & 6.428479 & -2.549715 & 0.149667 \\ \mathrm{~N} & -2.750864 & 1.634271 & -0.153842 \\ \mathrm{H} & 4.208442 & -2.658577 & -1.145706 \\ \mathrm{H} & 2.518749 & -0.856210 & -1.310587 \\ \mathrm{H} & 5.015732 & 1.698738 & 1.093024 \\ \mathrm{H} & 6.692407 & -0.125734 & 1.289902 \\ \mathrm{H} & 1.213057 & -0.513803 & 0.676696 \\ \mathrm{H} & -1.215342 & -0.512481 & 0.678789 \\ \mathrm{H} & -1.243931 & 3.634681 & -0.630967 \\ \mathrm{H} & 1.243391 & 3.633250 & -0.633471 \\ \mathrm{H} & -2.518945 & -0.849969 & -1.312662 \\ \mathrm{H} & 7.217447 & -2.296445 & 0.687773 \\ \mathrm{C} & -3.615185 & 0.542220 & -0.060786 \\ \mathrm{C} & -3.409524 & -0.698242 & -0.707005 \\ \mathrm{C} & -4.360048 & -1.709920 & -0.633817 \\ \mathrm{C} & -5.539302 & -1.519767 & 0.101293 \\ \mathrm{C} & -5.768761 & -0.287897 & 0.733635 \\ \mathrm{C} & -4.831952 & 0.732665 & 0.627059 \\ \mathrm{O} & -6.424983 & -2.552477 & 0.146354 \\ \mathrm{H} & -6.692005 & -0.131203 & 1.291951 \\ \mathrm{H} & -5.017603 & 1.695915 & 1.098644 \\ \mathrm{H} & -4.206076 & -2.655349 & -1.150658 \\ \mathrm{H} & -7.214155 & -2.301751 & 0.685353\end{array}$


B3LYP/6-31G* optimized structure of anti emeraldine hydroxyl terminated trimer in acetonitrile solvent.

Cartesian coordinates in Ångstroms.

$\begin{array}{rrrr}\mathrm{H} & -7.678401 & -1.712490 & -0.194008 \\ \mathrm{H} & -5.515977 & -2.159226 & -0.886394 \\ \mathrm{H} & -2.027146 & -1.141368 & 0.945217 \\ \mathrm{H} & 2.004405 & 1.285133 & -0.775035 \\ \mathrm{H} & -6.846192 & 1.568706 & 0.793844 \\ \mathrm{H} & -3.231990 & -1.264404 & -1.085358 \\ \mathrm{H} & 0.205564 & -2.124702 & 1.240376 \\ \mathrm{~N} & 2.418366 & -1.158839 & 0.487574 \\ \mathrm{C} & 1.300380 & -0.516253 & 0.276990 \\ \mathrm{C} & 1.130287 & 0.780308 & -0.380181 \\ \mathrm{C} & -0.096889 & 1.325793 & -0.541638 \\ \mathrm{C} & -1.322196 & 0.649621 & -0.120348 \\ \mathrm{C} & -1.151275 & -0.638719 & 0.551216 \\ \mathrm{C} & 0.076142 & -1.179297 & 0.721779 \\ \mathrm{~N} & -2.448523 & 1.263997 & -0.370343 \\ \mathrm{C} & -3.699719 & 0.663221 & -0.218665 \\ \mathrm{C} & -4.757907 & 1.462047 & 0.266155 \\ \mathrm{C} & -6.042933 & 0.955364 & 0.397044 \\ \mathrm{C} & -6.324182 & -0.358938 & -0.006344 \\ \mathrm{C} & -5.300067 & -1.151861 & -0.539330 \\ \mathrm{C} & -4.005406 & -0.649418 & -0.636428 \\ \mathrm{O} & -7.608850 & -0.797950 & 0.125210 \\ \mathrm{H} & 4.383445 & -2.507523 & -0.496156 \\ \mathrm{H} & -4.541552 & 2.485008 & 0.559219 \\ \mathrm{H} & -0.225217 & 2.275076 & -1.053479 \\ \mathrm{C} & 3.686316 & -0.620856 & 0.264318 \\ \mathrm{C} & 4.085217 & 0.686406 & 0.622646 \\ \mathrm{C} & 5.395227 & 1.111949 & 0.434408 \\ \mathrm{C} & 6.343045 & 0.250599 & -0.134000 \\ \mathrm{C} & 5.971499 & -1.058126 & -0.475747 \\ \mathrm{C} & 4.670181 & -1.489371 & -0.251082 \\ \mathrm{O} & 7.607174 & 0.730833 & -0.311254 \\ \mathrm{H} & 6.704822 & -1.739071 & -0.900250 \\ \mathrm{H} & 8.170616 & 0.039652 & -0.695908 \\ \mathrm{H} & 5.700831 & 2.111158 & 0.729569 \\ \mathrm{H} & 3.373334 & 1.355231 & 1.095753\end{array}$

